\title{
Assistive Diagnostic Tool in CT scans of Lungs anomalies by Utilizing Various Imaging Techniques
}

\author{
Saroosh Bilal ${ }^{1}$, Syed Omer Gilani ${ }^{1}$ and Mohsin Jamil ${ }^{2}$ \\ ${ }^{1}$ Department of Biomedical Engineering \& Sciences \\ ${ }^{2}$ Department of Robotics \& Artificial Intelligence \\ School of Mechanical \& Manufacturing Engineering (SMME) \\ National University of Sciences \& Technology (NUST) \\ Sector H-12, Main Campus, Islamabad, Pakistan \\ sarooshbilal.bms@smme.edu.pk,omer@smme.nust.edu.pk, \\ mohsin@smme.nust.edu.pk
}

\begin{abstract}
Lung cancer is one of the most prevalent causes of death among cancer patients. Later thee diagnosis is, lesser are the chances of recovery. However, patients have a higher chance of survival if it can be diagnosed in the primary stages. No one can reject the importance of CT, CT-PET \& PET modalities in the diagnosis of pulmonary problems. Modalities mentioned above have their own limitations. Digital Image Processing Techniques can be helpful in detection of lung nodule. Utilization of various imaging techniques is helpful in ruling out the area and type of anomaly. Contrast Adjustment, Segmentation, Thresholding of image are such techniques which enhance the acuity of the visibility of the defected region, however the feature extraction methodologies are helpful in ruling out the specificity of the defected region determining the extent of damage to the soft tissue structures. Utilization of such methods in routine clinical practices can serve as a helping hand to clinicians, as they will be able to take decision of treatment earlier with early detection of anomalies s. In this paper we developed such methodology which can be helpful in early detection and diagnosis of the presence on lung anomaly.
\end{abstract}

Keywords: Positron Emission Tomography (PET), computerized tomography (CT), Integrated Positron Emission Tomography and Computerized Tomography, lungs, tool

\section{Introduction}

The mortality rate among cancer patients is highest for lung cancer. The patient has low chances of survival after late diagnostics. Over the past decade there has been steady increase in deaths per year. This in part is due to late stage detection of the caner and continued growth even after late detection and treatment. However, the chances of patients to recover significantly improve if cancer is diagnosed in early stages. This leads to proposal of an automated system that can assist in early diagnostic of such cases.

\subsection{Classification}

We can make two broader classes of Lung cancer as

1. Non-small cell lung cancer

2. Small- cell lung cancer.

Cellular features are the foundation of this classification.

Received (June 23, 2017), Review Result (September 28, 2017), Accepted (October 5, 2017) 


\subsection{Staging}

Stages of lung cancer are four in number, I through IV based on

- Tumor size

- Tumor locality

- Lymph node location.

\subsection{Modalities Use for Diagnosis}

No one can repudiate the importance of CT, CT-PET \& PET modalities in the diagnosis of pulmonary problems [4]. Though, it is found out that CT scan can fairly reveal the morphological variations in the region but has limitations in depicting the begnin or malignant nature of lesion if it is considered for detecting pulmonary oncology. For this purpose Positron Emission Topography (PET) is considered as a reliable source. Combining both of the modalities will reveal the most accurate results [5]

Mediastinal lymph-node metastases \& also the extrathoracic metastases are fairly detected by whole-body positron emission tomography (PET) with uptake of a radioactive glucose solution [5]. Besides oncological pulmonary problems, the identification of nononcological pulmonary lesion and lung nodule growth and size can be fairly determined by taking into consideration the utilization of various imaging techniques which can be helpful for the radiologists and clinicians to determine the regional probability of certain lesions and also able to define the probable area of increased activity.

In our study, we are finding out that how probability to define the region optimal for anomalies; can be increased by utilizing various imaging techniques, when CT, integrated CT- PET \& PET alone are taken from different sites and angles.

Small lung nodules are a common radiographic finding which is proven as an important diagnostic encounter in contemporary medicine. As pulmonary nodules are the major radiographic pointer of lung cancer, they may also be symbols of a variety of benign disorders. Not only in diagnosis of the anomalies, distinguishing malignancy and non-malignancy in real time is also helpful to plan the future treatment of patient accordingly [11].

\subsection{Certain Limitations of Modalities}

CT scan has its own limitations with \&/or without contrast so as the PET as commercially available PET scanners provide nominal spatial resolution within the resolution of 4.5 to $6.0 \mathrm{~mm}$ in the central region of axial field of view therefore it is made possible that lesions which are even $<1 \mathrm{~cm}$ with respect to diameter can be detected on the basis of an increased uptake of radioactive glucose solution. But one should also consider the fact that PET offers indefinite evidence on the exact site of central irregularities [5].

\section{Methodology}

Procedure involved in the lung cancer finding system for this study can be viewed in Figure 1.

\subsection{Collection of Dataset}

For this study, data set of CT, PET and integrated PET- CT is taken from the online available image repositories at National Biomedical Imaging Achieve arranged by National Cancer Institute USA in the dicom (.dcm) format [9]. In this study, we have taken datasets for ten different subjects and applied image processing techniques via MATLAB to find out that in which region the probability of finding lesion is 
comparatively more than the other by improving image quality by utilization of different methods.

\subsection{Criteria for Selection of Dataset}

The criteria of selection of dataset was the anatomical regions (restricted to chest and lungs), with lesion of uncertain extent. Demographics of patient and other clinical histories are not considered in this study. CT images were taken from either coronal plane or sagittal plane with or without contrast and PET images were either axial or non-axial (Table: 1).

\subsection{Image Processing Methods}

Nevertheless, PET is a reliable means of defining the over-all site of a tumor focus, its capability to outline accurately the geometric size of such a focus strictly depends upon choice of a suitable image threshold. However, in clinical practice, the image threshold is usually not selected according to consistent, well-established criteria [2]. Moreover, we will also consider the effect of intensity variations (gradient differences between the foreground and background) as it is seemed to improve the visual quality of image [3].

\section{Image Acquisition}

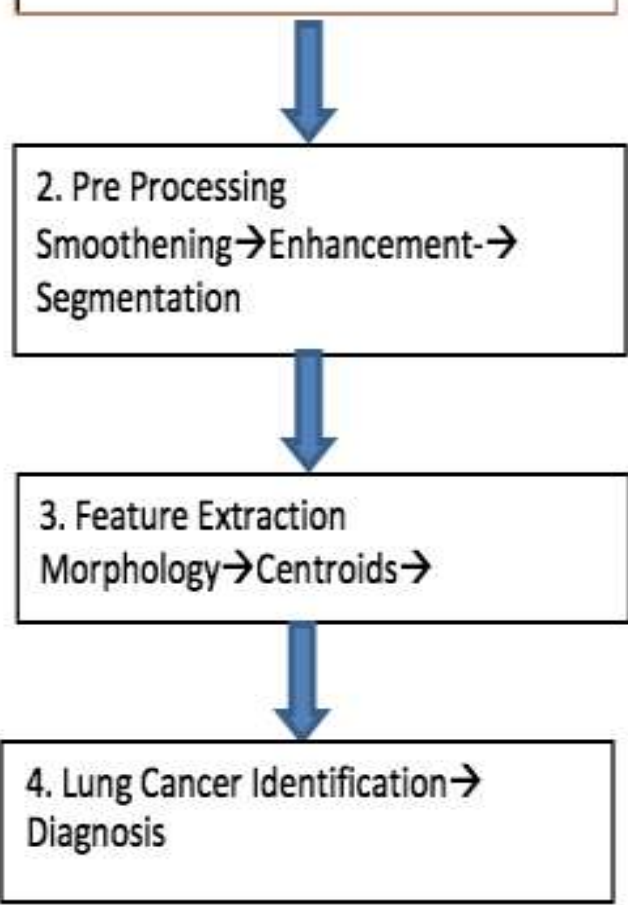

Figure 1. The Proposed System Block Diagram 
Table 1. (Data Set is Taken From Online Available Image Repositories at National Biomedical Imaging Achieve arranged by National Cancer Institute USA)

\begin{tabular}{|c|c|c|c|c|c|c|c|}
\hline 2 & A & 3 & c & o & E & $F$ & to \\
\hline $1 \%$ & Patient id & Study Deseriptien & Medalit & YSeries Description & Manufacturef & Manufaturet Medel & Seftware Versien \\
\hline 26 & CSMHAO4 & CTCHEST W/OCONTLST & $\pi$ & $\cos$ & all & nul & 250 \\
\hline 38 & csumsoss & CT CHEST W/O CONTFAST & a & WNS & null & sul & 250 \\
\hline 4 & camialas & CTCHEST W/O CONTEAST & ऑा & NCencor & null & sult & 250 \\
\hline 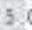 & GSMnLsou & CTCHEST W/O CONTRAST & $\pi$ & sea & nul & $s i$ & 250 \\
\hline 6.8 & cosproses & VE/KT Ling Concet & $\pi$ & ctimages & GF WEDKCAL SFStEMS & ת4 & 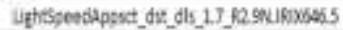 \\
\hline 70 & cosnrose4 & FET/CTLuncascer & शि & FEा BODE CTAC & G. VEDKALALSMTEMS & nul & \\
\hline 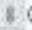 & GSW710044 & VEikt tung Concer & Pr & IE_BOOH_NO_AC & G: NEDKALISPTISUS & sull & \\
\hline 98 & somnsos & Thorarv1 NowcowChEs [adsit)] & a & Thoras $10845 f$ & nell & sel & sngoct 2000 s \\
\hline 100 & Gsantaves & Poravon__NCEVCONCHEST (Add) & a & Therar $20 \$ \$ 0$ oor & sult & sult & snesctrans \\
\hline II & 65M710065 & 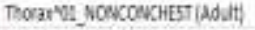 & $\pi$ & Thorar $20590 \mathrm{sg}$ & mull & nul & sngect 200Ts \\
\hline 12 & GsMniates & PoraxVI_NOWCONCHEST (Adutt) & $\pi$ & Toras 5.083 if & tult & sull & sngoct $200 x$ \\
\hline 11 & GSM7LS045 & 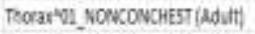 & $\pi$ & Thorax $7.0 \mathrm{MP}$ ax & nd & null & sygect 200Ts \\
\hline 146 & GSMTIA065 & Tharavn_sCWCOWCHEST (Ad_It) & $\pi$ & Tepceram 5.6 T205 & sult & nd & snąctraxs \\
\hline 150 & CSMP10N5 & setict Luey Carcer. & $\pi$ & cTingeges & GE NEDKCAL 5PSTEM5 & sul & 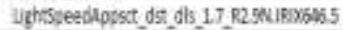 \\
\hline 15. & Gantians & FEt/CTling Cincer & मा & IE_ BODF_CTAC & GE VEDKCAL SYSTEMS & sul & \\
\hline I7 & CQMTLSOS5 & PER/TTLung Cancer & pT. & SET BOOY NOAC AC & जE MEDICAL S/STMS & sul & \\
\hline 186 & GSMTA05 & CTCHEST W/O & $\pi$ & abmincet & G: NEDICAL SESTEMS & Desouery CTSSO & anws9. 4 \\
\hline B) & GSM715065 & CTCHEST W/O & a & Coronals: & G. MEDKCAL STSTEM 5 & $0 \sin x$ & ognwase \\
\hline 20 & CSMT10045 & PE imaGe Wilt, stall & at & CT_SLCES & GE VEDICALSNSTENS & Disoutry STE & 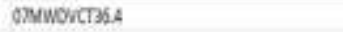 \\
\hline as & CoMn1506\% & РET IMAGE W/CT, 5RML- & PT & AXILE PET & GE MEDKALISTTEV5 & Dosweryst: & \\
\hline ne & 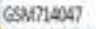 & CT ANGOSALAT, OEST & ๘ & Q.65 DUPA 0n +5550 & DGF WEDKCAL SYSTEMS & Dsebuery CTSOHO & CaMW3.4. \\
\hline 86 & ESMTINon? & CT NWSOGRAPH, DEST & $\pi$ & Coronats & G: VEDIKAL.SPSTEM & $\cos x$ & anw394 \\
\hline $24:$ & CSMTLAST & 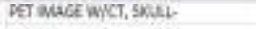 & का & C__ules & GE MEDICN SHSTEMS & ancownste & 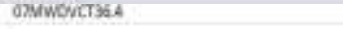 \\
\hline a. & Cannos? & PET muce wict, suKL- & \# & AnAL मET & GE Midical sstems & Osxoveryste & \\
\hline 280 & G9Mnas45 & CTCHEST ABD PEIVS WTH CON & $\pi$ & Carouls 0.69m & nall & $\operatorname{cosin} x$ & șyoc 020005 \\
\hline $2 \pi 0$ & esanianes & CTCHEST ABO VEI WS WTH CON & at & THCARX10345t & nall & nil & sngact 2000 s \\
\hline 18. & Canprosa & 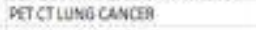 & a & CTinags & GE METrCue SHSTans & nall & 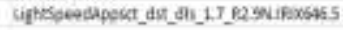 \\
\hline as & GoMnases & PETCTUNGCANCa & st & PEt_ac0 _CT 25 & GE MEOCAC SMIEMS & nal & \\
\hline 100 & GSMTrosua & PETCT UNG CANCRH & $\pi$ & Per_BCO N NO $A \mathrm{~K}$ & GE MEDCAL SYSTEKS & nail & \\
\hline 31 & 6SM7140049 & CT THOSAX & $\pi$ & OESTLZSMM & mall & nal & aMmist \\
\hline 12. & 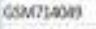 & ctomosux & aा & Cemanaiso.somm & natl & $\operatorname{oun} x$ & ganwise \\
\hline a. & Gornases & PET CT LUNG CANCER & $a$ & Cringest & Ge Medicus Srsteus & null & 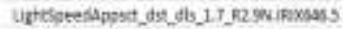 \\
\hline 34 & esminave & PETCTUKGG CANCER & $\pi$ & PEE, $\mathrm{BOOK}$ СTAC & GE MEDCCL SYSTEMS & mil & \\
\hline 350 & -6SM752049. & DET CT LUNE CENLER & भा & DET_aCOY_NO_AC: & GE MEEDCCL SYSTEMS & nall & \\
\hline \#c & conneaso & croutsi wio & ct & Comati & er miocicussstems & Ounix & 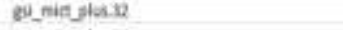 \\
\hline IIi & CSMMrano & तCHEST W/O & $\pi$ & THIV LUNG WINDOW & GE MEOCLL SYSTEVS & Quscovery CTrY top & Di_mitylos 32 \\
\hline 380 & Esannasso & PET AMUEE W/KT, SNUCL. & er & C) SULES & GEMEOCAL STSTEMS & Ascowery STE & dince, ductupl 13 \\
\hline 19. & csoniatso & 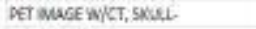 & or & AXEALET & GE NEOCAL SYSTEMS & OtCOCWENYSTE & \\
\hline 40. & cennoss! & ccuss w/o & $a$ & . 225 mnchest & Ge meacus ststevs & Desonetyctossive & atuwes \\
\hline 43 & Gsannwosi & तCHEST W/O & $\pi$ & Coronts & GE MeOCLU SVSTEMS & $\operatorname{cosin}$ & OSWWBSA \\
\hline $43: 5$ & GSMTLA251 & PET MUGE W/KT, SWOLL & ct & CT SULE & GE MEOCLA SYSTEMS & OSxowery STE & GMMWVUCT3L 4 \\
\hline 45. & Gsantiatsi & PET MUGE WikT, SKCEL & or & AXALBET & GE MEDECOL SYSTENS & Diecoweryste & \\
\hline ai & CQMThas? & ctoutst wiान & s & Comanh & Gi MtoCA STSTIMS & aurix & gi_nitysing \\
\hline as: & GsMrinass & CTCHE⿱T⿰㇀丶㇀ & $\pi$ & THEX LUNG WINDOW & GEMEDICL SYSTEVS & OScovery CTMOHO & setrictias 32 \\
\hline $16 \mathrm{C}$ & CSMM7LaE52 & PET AMASEW/CT, SNOLL & ar & CT.2.TES & GE MEDCCAL SYSTEMS & CiscoverysTe & GMWWDVtT34 \\
\hline 476 & GSM71452 & PET MAGE WICT, SXUL- & PT & AXALPET & GEMEDICAL S1STEUS & Oiscovery STE & \\
\hline $48.8-2 \cdot 3-1$ & 65M714051 & CTCHEST & CT & .625 mmchest & GE MEXCAL SYSTEMS & Oiscoveny (7750 HD & Q8MN39.A \\
\hline 436 & 65M714053 & CTCHEST & ¿T & Coronals & GEMEDICAL SISTEMS & $0 \sin x$ & COMNA35.4 \\
\hline 508 & GSM714563 & PET IMAGE Wilt, UNTL & CT & đT_SUCES & GE MEQUCAL SISTEMS & Discoveny STE & OPMADUCT36.4 \\
\hline 51 & 6SM714553 & PET MAGE WITT, UMTD & PT & AXALPET & GEMEDICAL SYSTEMS & Ciscoveny STE & \\
\hline
\end{tabular}

\subsection{Pre Processing Techniques}

As there may be the presence of marks (Film artifacts) which can interfere in the postprocessing of images, they are henceforth; needed to be made more suitable for further processing in system. Noise in the image also needs to be removed to avoid any missed diagnosis or mal diagnosis. Here an attempt has been made to identify the suitable algorithms for pre processing the images having lesion (lung region)[12].

To improve image quality, the degree of interpretation or acuity of information in images for a viewer, or to provide 'better' input for other automated image processing techniques; Image enhancement is considered one of the most important parameter.

For that reason, preprocessing process was carried out to ensure above mentioned parameters. Image pre-processing methods considered in this project are smoothing, enhancement, and segmentation [7]. 


\subsection{Image Enhancement}

As we know that image enhancement utilizes: Spatial domain methods and frequency domain methods. When it comes to visual acuity, inopportunely, there is no broadspectrum principle for determining what "worthy" image enhancement is. If it looks good, it is good

For low contrast (CT, CT-PET or PET) images contrast is increased and then segmentation is applied to rule out the presence of abnormality. The segmentation of images with high resolution is typically easier as compared to images with lesser resolution on account of border definition difficulties. For normal contrasted images segmentation is directly applied to define the region where nodules or tumor growth is apparent [8].

\subsection{Image Segmentation}

Image segmentation is a crucial method for most image examination succeeding tasks. In precise, many of the present techniques for image depiction and recognition depend greatly on the segmentation consequences. Delineation of anatomical structures thus carried by the segmentation method of the image processing is important from in diagnosis of anomalies with in the pulmonary region primarily malignant $\&$ or malignant nature of the lesions.

In this study we utilized following methods for segmentation:

\section{Thresholding Based Segmentation}

\section{Histogram Based Segmentation}

\subsection{Thresholding based Segmentation}

It is a powerful technique for image segmentation. The segmented image attained from Thresholding has the benefits of lesser storing space, fast processing speed and comfort in manipulation, compared with gray level image which usually contains 256 levels. It is easy to detect a cell using edge detection and basic morphology. Demarcated cell can be easily identified in an image if the object has sufficient contrast from the background [1].

\subsection{Histogram based Segmentation}

It is relatively trivial but effect technique. In this technique pixels are grouped in such a way that they label different objects and regions in the image. In typical image the intensity values range between 0-255 integer values (for 8 bit image intensity encoding). A histogram of these values results in a distribution with many peaks and valleys. These identify different regions/objects in the image and are useful for segmentation of region/object of interest.

\subsection{Feature Extraction Based Techniques}

Feature extraction based techniques are relatively complex yet powerful. To detect and isolate various desired regions or shapes (features) of an image showing significant results to determine the regularity or irregularity within an image by using different parameters [6]

In this study following features were considered; mean intensity, area, eccentricity (roundedness), centroids, and perimeter.

Area: represented by a scalar value which indicates the area of lung nodules. The area is calculated by summing all pixels of the nodule, which are labeled as 1 in binary image.

Perimeter: represented by a scalar value which indicates the boundary of lung nodule. It is calculated by adding all the pixels that connect the boundary line encompassing the nodule. Here boundary pixels are labeled as 1 in binary image. 

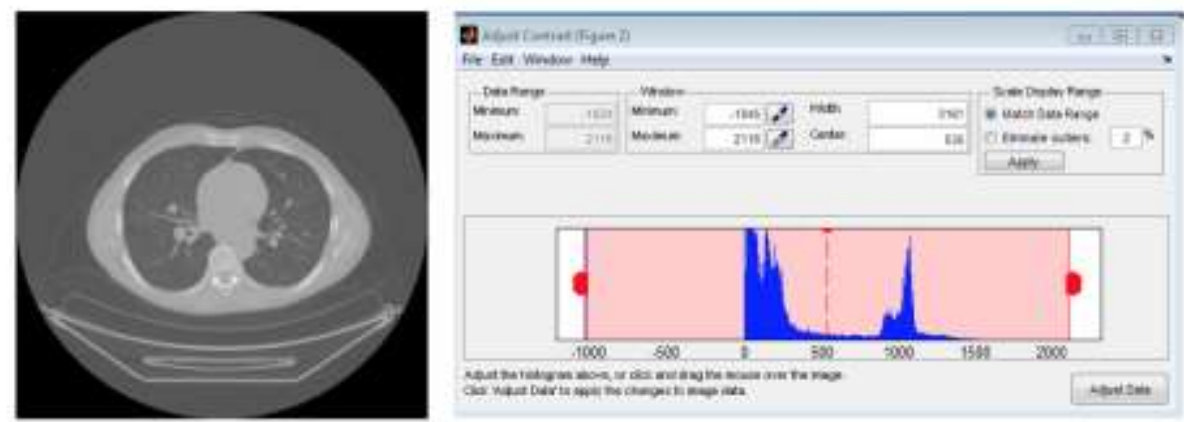

(A)
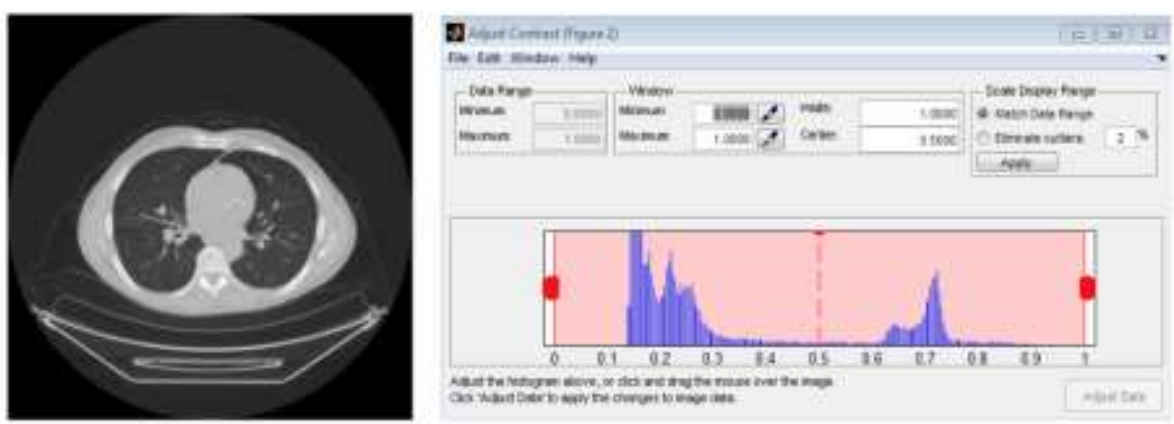

(B)

\section{Figure 2. (A) Original Image from the Database. (B) Contrast Enhanced Image. The Results are also Shown Using Pixel Intensity Distribution (Histogram)}

Eccentricity: It is the measure of roundedness depicting circularity or irregularity. A value one (1) is assigned to perfectly circular shape while a value of less than one $(<1)$, is assigned to other shapes.

Centroid: It is the measure of center of gravity of an object. In n-dimensional space, it is the mean position of point in all every coordinate direction.

\section{Results}

As mentioned in methodology section above, the first step in any image processing based system is to pre-process the image to make more convenient for use by automated algorithms.

Figure 2 shows the image preprocessing step. The example is taken from image repository hosted at Department of health services NIH NCI. Here we show the original and transformed image along with their histograms (pixel intensity distributions). The top row shows original image while bottom row shows contrast enhanced image. As evident by pixel intensity distribution (shown in right for both $\mathrm{A}$ and $\mathrm{B}$ ), image contrast has significantly improved both qualitative and quantitative results. 

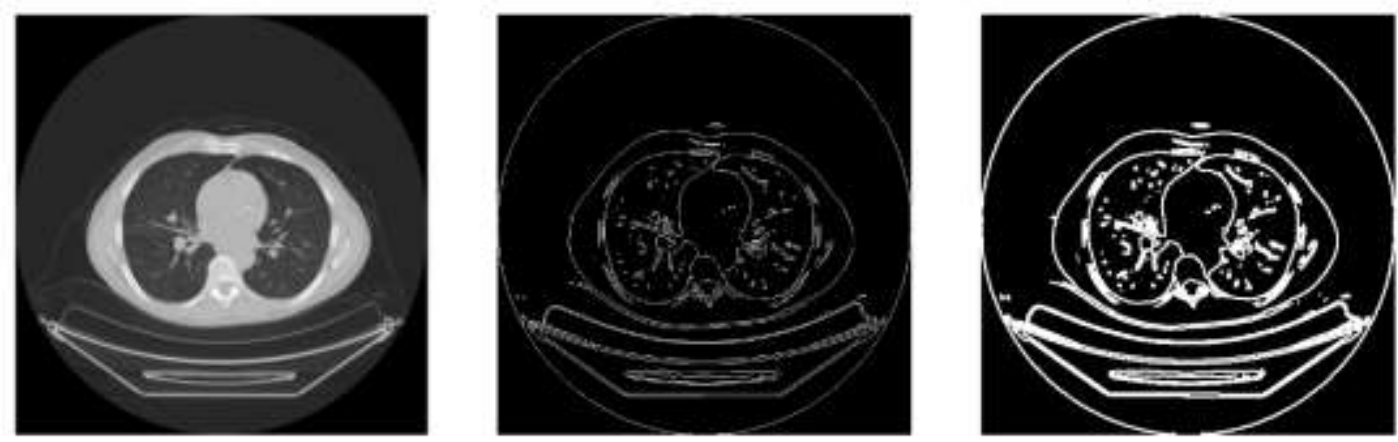

\section{Figure 3. (left) Contrast Enhanced Image (middle) Thresholding to Identify The Regions (Right) Segmented Regions using Morphological Operations and Connected Component Analysis}

Contrast enhanced image is processed through thresholding algorithm. An adaptive threshold technique is used to remove unwanted background area (tissue region) and leaving only structural information (Figure 3 middle). A processed image is then subjected to morphological operations (Figure 3 right). The image is first dilated to fill the gaps in close regions that were disconnected due to thresholding. This results in similar regions/object connected by pixels thus reflecting a coherent region/object. As shown the regions they are now well connected. This is followed by connected component analysis to isolate and label different regions/objects in the image.

Interestingly histogram based segmentation technique also leads to good segmentation. As shown in Figure 4, different histogram regions selection enables visibility of different regions. An intelligent algorithm can utilize different combination of these images (like and, or, xor) to isolate the desired regions/objects
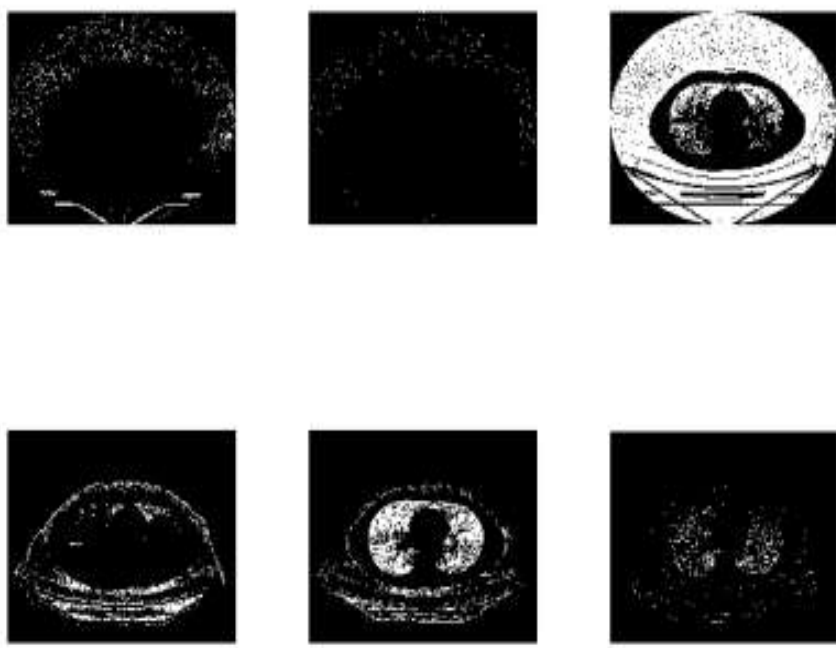

Figure 4. Results of Histogram based Segmentation

Limitations: Although the aforementioned procedures are appropriate in defining the presence of anomalies more appropriately but there exists limitations of above mentioned procedures. Though these techniques are able to provide great visible acuity of the image with a lesser noise but the extent of lesion can't be yield by it. Moreover, the accuracy of image in detecting and defining the lesion is promising one but the type of lesion can't be find out. 
Another limitation in working with the purposed methodology is that we don't know whether it will work or not when images of region other than those containing soft tissues is the region of interest.

\section{Conclusion and Discussion}

Utilizing the basic image processing techniques enables the viewer to increase visibility and to check out fine details of the image with maximum ease. To attain more precise outcomes, we developed our work into three stages: Image Enhancement, Image Segmentation and Features Extraction. Detection of lung nodule is an active area of research which is continuously emerging and there are many augmentations that can be encompassed to make it more resourceful. When we consider the forthcoming of utilization of these methods, we concluded that it is a possibility to generate a code which will act as an automated diagnostic tool and will act as the most effective aid in automated diagnosis and classification of Lung anomalies. In such an automated diagnostic tool, we can make our machine to learn about the differences between various lesions based on parameters (area, perimeter, centeriod location, and average intensity). And it will help clinicians to rule out anomalies easily and more accurately.

Future Prospects: Further room of research is available to make an algorithm which not only provides increased visibility but also be able to define the configuration as well as classification of specified lesion. Segmentation of the whole lung can be carried out from the CT images and the parameters are calculated from the segmented image. As we know through previously done work that the statistical parameters like mean, skewness, standard deviation, kurtosis, fifth central moment and sixth central moment are used for classification [10].

Henceforth a new image processing technique which can be able to work on the above mentioned parameters can be thus devised which provides the practitioner with a an image which is having a clear vision, highlighting all the major and minor details and also be able to classify the type of anomaly.

\section{References}

[1] A. Chaudhary and S. S. Singh, "Lung Cancer Detection on CT Images by Using Image Processing", 2012 International Conference on Computing Sciences, (2012).

[2] Y. E. Erdi, "Segmentation of lung lesion volume by adaptive positron emission tomography image thresholdin.", Cancer, vol. 80, no. S12, (1997), pp. 2505-2509.

[3] H. Zaidi, "Comparative methods for PET image segmentation in pharyngolaryngeal squamous cell carcinoma", European journal of nuclear medicine and molecular imaging, vol. 39, no. 5, (2012), pp. 881-891.

[4] T. I. E. L. C. A. P. Investigators, "Survival of Patients with Stage I Lung Cancer Detected on CT Screening", New England Journal of Medicine, vol. 355, no. 17, (2006), pp. 1763-1771.

[5] R. L. Wahl, "Staging of mediastinal non-small cell lung cancer with FDG PET, CT and fusion images: preliminary prospective evaluation", Radiology, vol. 191, no 2, (1994), pp. 371-377.

[6] K. Suzuki, "False-positive Reduction in Computer-aided Diagnostic Scheme for Detecting Nodules in Chest Radiographs by Means of Massive Training Artificial Neural Network", Academic Radiology, vol. 12, no 2, (2005), pp. 191-201.

[7] El-Baz, A. A. Farag, R. Falk and R. L. Rocco, "detection, visualization, and identification of lung abnormalities in chest spiral CT scans: phase I", Information Conference on Biomedical Engineering, Egypt, (2002).

[8] Hadavi, M. Nordin and A. Shojaeipour, "Lung cancer diagnosis using ct-scan images based on cellular learning automata", in Computer and Information Sciences (ICCOINS), 2014 International Conference on. IEEE, (2014), pp. 1-5

[9] Online Medical image Repositories by Department of Health and Human Services | National Institutes of Health | National Cancer Institute | USA.gov.

[10] J. Kuruvilla and K. Gunavathi, "Lung cancer classification using neural networks for CT images", Computer methods and programs in biomedicine, vol. 113, no. 1, (2014), pp. 202-209.

[11] W. J. Kostis, A. P Reeves, D. F. Yankelevitz and C. I. Henschke, "Three-dimensional segmentation and growth-rate estimation of small pulmonary nodules in helical CT images", IEEE transactions on medical imaging, vol. 22, no. 10, (2003), pp. 1259-1274. 
[12] S. Patil and V. R. Udupi, "Preprocessing to be considered for MR and CT images containing tumors", IOSR journal of electrical and electronics engineering, vol. 1, no. 4, (2012), pp. 54-57.

\section{Authors}

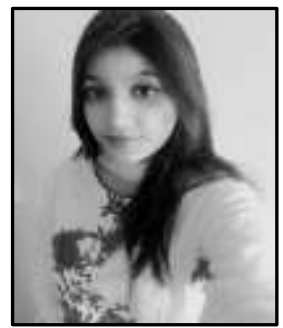

Saroosh Bilal, he is currently Masters Student at Department of Biomedical Engineering and Sciences within NUST School of Mechanical and Manufacturing Engineering (SMME). Her research interests are in the field of biomedical sciences.

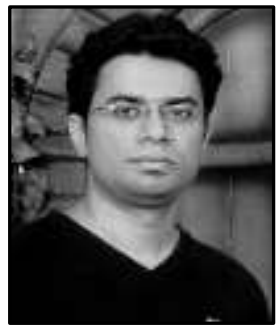

Syed Omer Gilani, he is currently an assistant professor at National University of Sciences and Technology (NUST), Pakistan. He earned his Ph.D. in electrical and computer engineering from National University of Singapore in 2013 and MSc degree in computer engineering from Sweden in 2006. Between 2006 and 2008, he worked at Interactive Multimedia Lab (Singapore). His research interests include human-machine interaction and networking and actively consults for industry on various projects.

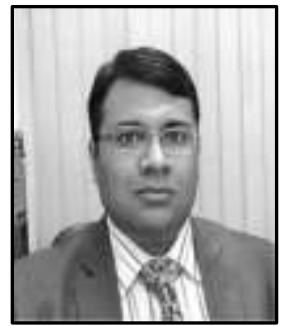

Mohsin Jamil, he is currently assistant professor and Dy Head of Department at National University of Sciences and Technology (NUST), Islamabad, Pakistan. He received his BEng Industrial Electronics degree from NED University, Pakistan, in 2004. He is MSc Electrical Engineering degree holder from Dalarna University Sweden and National University of Singapore in controls and automation. He received $\mathrm{PhD}$ Degree from University of Southampton, UK. His research interests include digital control design and smart grid technologies. He is author of several IEEE publications. 
International Journal of Signal Processing, Image Processing and Pattern Recognition Vol. 10, No. 12 (2017) 